\title{
烧结方式对 $(\mathrm{K}, \mathrm{Na}, \mathrm{Li})(\mathrm{Nb}, \mathrm{Sb}, \mathrm{Ta}) \mathrm{O}_{3}$ 压电陶瓷的 微观结构和物理性能的影响
}

\author{
张晓晨 ${ }^{1}$, 王雪梅 ${ }^{2}$, 王春雷 ${ }^{2}$ \\ (1. 四川大学 物理科学与技术学院, 成都 $610065 ; 2$. 山东大学 物理学院, 济南 250100)
}

摘 要: $(\mathrm{K}, \mathrm{Na}) \mathrm{NbO}_{3}$ 基陶瓷(KNLNST 陶瓷)是一类很有发展潜力的无铅压电材料, 目前对其进行两步烧结相关的研 究还很少。本工作分别采用普通烧结(Conventional Sintering, CS) 和两步烧结(Two-step Sintering, TSS)制备了 $\left(\mathrm{K}_{0.4425} \mathrm{Na}_{0.52} \mathrm{Li}_{0.0375}\right)\left(\mathrm{Nb}_{0.8825} \mathrm{Sb}_{0.08} \mathrm{Ta}_{0.0375}\right) \mathrm{O}_{3}$ 陶瓷, 并进行微观结构与物性的对比研究。 $\mathrm{TSS}$ 可将 $(\mathrm{K}, \mathrm{Na}) \mathrm{NbO}_{3}$ 基陶瓷 的相对密度 $\rho^{\prime}$ 由 $\mathrm{CS}$ 时的 $95.0 \%$ 提高至 $97.0 \%$, 压电系数 $d_{33}$ 由 $\mathrm{CS}$ 时的 $363 \mathrm{pC} / \mathrm{N}$ 增大到 $387 \mathrm{pC} / \mathrm{N}$ 。两种烧结方式 制备的 KNLNST 陶瓷的微观组织结构和电畴结构有着很大的差异。KNLNST-CS 陶瓷的晶粒尺寸较小而且分布较 为均匀, 极化后多数晶粒的电畴图案为简单的平行条纹。KNLNST-TSS 陶瓷的晶粒尺寸则大小分布不均匀, 极化后 许多大晶粒中呈现带状条纹内部又存在着精细的平行条纹的电畴图案。

关 键 词: $(\mathrm{K}, \mathrm{Na}) \mathrm{NbO}_{3}$ 基陶瓷; 两步烧结; 压电性能; 相变; 微观组织结构; 电畴结构 中图分类号: TQ174 文献标识码: A

\section{Influences of Sintering Methods on Microstructure and Physical Property of (K,Na,Li)(Nb,Sb,Ta) $\mathrm{O}_{3}$ Piezoelectric Ceramics}

\author{
ZHANG Xiao-Chen ${ }^{1}$, WANG Xue-Mei ${ }^{2}$, WANG Chun-Lei ${ }^{2}$ \\ (1. College of Physical Science and Technology, Sichuan University, Chengdu 610065, China; 2. School of Physics, Shandong \\ University, Jinan 250100, China)
}

\begin{abstract}
K,Na) $\mathrm{NbO}_{3}$-based ceramics (KNLNST ceramics) are a very promising type of lead-free piezoelectric materials. However, only limited studies were reported on them prepared by two-step sintering. Here, a comparison study was performed between two kinds of $\left(\mathrm{K}_{0.4425} \mathrm{Na}_{0.52} \mathrm{Li}_{0.0375}\right)\left(\mathrm{Nb}_{0.8825} \mathrm{Sb}_{0.08} \mathrm{Ta}_{0.0375}\right) \mathrm{O}_{3}$ ceramics that were prepared by either conventional sintering (CS) or two-step sintering (TSS). It was found that TSS largely increases the relative density $\rho^{\prime}$ and piezoelectric coefficient $d_{33}$ from $95.0 \%$ and $363 \mathrm{pC} / \mathrm{N}$ of $\mathrm{CS}$ to $97.0 \%$ and $387 \mathrm{pC} / \mathrm{N}$, respectively. KNLNST ceramics prepared by two kinds of sintering methods show significant differences between microstructure and domain structure. The KNLNST-CS ceramic has small grain sizes but distributed uniformly, and their domain patterns in most grains are simply a group or groups of parallel stripes. In contrast, the KNLNST-CS ceramic has a non-uniform grain-size distribution, with many large grains displaying broad bands, and existing fine strips.
\end{abstract}

Key words: $(\mathrm{K}, \mathrm{Na}) \mathrm{NbO}_{3}$-based ceramics; two-step sintering; piezoelectric property; phase transitions; microstructure; domain structure

收稿日期: 2018-09-20; 收到修改稿日期：2018-11-13

基金项目：国家自然科学基金(51672159) National Natural Science Foundation of China (51672159)

作者简介: 张晓晨(1997-), 女，本科生. E-mail: zhangxiaochen166@163.com

通讯作者: 王春雷，教授.E-mail: wangcl@sdu.edu.cn 
压电陶瓷是一类能够实现机械能与电能之间相互 转换的重要功能材料, 可用于制造超声换能器、滤波 器、驱动器、传感器等电子元件和器件, 在国民经济、 科学技术、现代国防等各领域有着广泛的应用。随着 社会对环境保护和可持续发展意识的日益增强, 世界 各国近年相继出台了各种相关的法规法令, 目前广泛 使用中的以 $\mathrm{Pb}(\mathrm{Zr}, \mathrm{Ti}) \mathrm{O}_{3}$ 为代表的含铅氧化物陶瓷(统 称为 PZT 陶瓷)因组分中含有大量的铅成分而被限制 使用。因此, 探索和发展可以替代 PZT 陶瓷的无铅压 电陶瓷材料是一项现实且迫切的重要任务。

铌酸钾钠基陶瓷被认为是一类非常有希望可以部 分替代 PZT 陶瓷的无铅压电材料。(K, $\mathrm{Na}) \mathrm{NbO}_{3}$ 陶瓷(简 称为 KNN 陶瓷)在室温下的晶体结构为正交晶相, 压 电性能较低, 利用通常生产电子陶瓷材料的工艺方法 制备的样品的压电系数 $d_{33}$ 和平面机电耦合系数 $k_{\mathrm{p}}$ 分 别为 $125 \mathrm{pC} / \mathrm{N}$ 和 0.41 左右 ${ }^{[1]}$ 。针对 $\mathrm{KNN}$ 陶瓷的压电 性能偏低的问题, 国内外研究者在过去十几年中从组 分改性的角度付出了大量的努力, 使得 $\mathrm{KNN}$ 基陶瓷 目前终于达到了可与 PZT 陶瓷相脑美程度的 $d_{33}$ 值。 有关提高 KNN 陶瓷的压电性能的组分改性的研究大 致可以分为两个阶段。在早期阶段, 多数研究主要采 取了以 $\mathrm{Li}$ 元素部分替代 $\mathrm{K}$ 元素和 $\mathrm{Na}$ 元素、以 $\mathrm{Ta}$ 元 素和 $\mathrm{Sb}$ 元素部分替代 $\mathrm{Nb}$ 元素的方法, 使正交-四方相 变温度 $T_{\mathrm{O}-\mathrm{T}}$ 降低至室温附近, 从而利用各种物理量于 相变点时呈现极值的特点在室温下获得较高的压电性 能 ${ }^{[1-6]}$ 。近年的许多研究则采取了向 $(\mathrm{K}, \mathrm{Na}) \mathrm{NbO}_{3}$ 或 $(\mathrm{K}, \mathrm{Na})(\mathrm{Nb}, \mathrm{Sb}) \mathrm{O}_{3}$ 中添加 $\mathrm{BaZrO}_{3} 、(\mathrm{Bi}, \mathrm{Na}) \mathrm{ZrO}_{3}$ 等氧化 物的掺杂方法, 使 $T_{\mathrm{O}-\mathrm{T}}$ 降低至室温附近, 同时使三角正交相变温度 $T_{\mathrm{R}-\mathrm{O}}$ 也提升至室温附近, 从而在室温下 获得更高的压电性能 ${ }^{[7-12]}$ 。

研究者们曾对利用热压烧结制备的 KNN 陶瓷与 普通烧结所制备的 $\mathrm{KNN}$ 陶瓷进行比较, 认识到致密 度对于压电性能有很大影响 ${ }^{[13]}$ 。由于 $\mathrm{Na}_{2} \mathrm{O} 、 \mathrm{~K}_{2} \mathrm{O}$ 等具 有挥发性, 为了避免烧结时这些碱金属氧化物成分的 挥发, 需要尽可能地降低烧结温度, 因而利用普通烧 结通常很难获得高致密度的 $\mathrm{KNN}$ 基陶瓷 ${ }^{[14]}$ 。虽然利 用热压烧结和放电等离子体火花烧结等方式可以提高 $\mathrm{KNN}$ 基陶瓷的致密度度、得到良好的压电性能 ${ }^{[13,15-16]}$, 但烧结设备比较昂贵, 不可避免地大幅度增加制备成 本，因而不太适合陶瓷材料的规模化生产。与前述的 这些烧结方式相比较, 两步烧结方式是一种获得晶粒 尺寸小、致密度高的陶瓷的现实而且有效的手段, 曾 被用于制备 $\mathrm{Y}_{2} \mathrm{O}_{3} 、 \mathrm{BaTiO}_{3}$ 等氧化物陶瓷材料 ${ }^{[17-19]}$ 。与 普通烧结方式相比, 两步烧结方式只是在温度随时间 变化的程序设置方面有一些区别, 不需要特殊的烧结 装置。对于一种成功的两步烧结而言, 第一步烧结程 序应将温度设置于一个较高值, 使拟烧结的成型体在 第一步烧结程序结束时达到中等程度的相对密度; 然
后, 迅速地将烧结温度降低至一个较低值, 在该温度 下进行长时间的保温，使烧结体的粒径尺寸不发生明 显的增大但相对密度得到显著的提高, 最终获得晶粒 尺寸小而致密度高的陶瓷材料。但是, 如前所述, 由于 KNN 基陶瓷的碱金属氧化物成分在高温下比较容易 挥发, 到目前为止, 利用两步烧结制备 KNN 基陶瓷的 研究报道还很少, 而关于两步烧结制备的 KNN 基陶瓷 的电畴结构的研究报道则更少 ${ }^{[20-23]}$ 。

综上所述，探讨两步烧结方式制备高致密 KNN 基陶瓷对于进一步发展 $\mathrm{KNN}$ 基无铅压电陶瓷材料、认 识相关的物理现象与规律, 具有重要的技术价值和科 学意义。本研究分别采用普通烧结方式和两步烧结方式 制备了组分为 $\left(\mathrm{K}_{0.4425} \mathrm{Na}_{0.52} \mathrm{Li}_{0.0375}\right)\left(\mathrm{Nb}_{0.8825} \mathrm{Sb}_{0.08} \mathrm{Ta}_{0.0375}\right) \mathrm{O}_{3}$ 的陶瓷(以下简称为 KNLNST 陶瓷)，从晶体结构、相 变、微观组织结构、电畴结构和压电性能等方面进行 了详细的对比，研究烧结方式对诸种物性的影响。

\section{1 实验方法}

实验采用了分析纯度的 $\mathrm{K}_{2} \mathrm{CO}_{3}$ (上海国药, $\geqslant$ $99.0 \%$ )、 $\mathrm{Na}_{2} \mathrm{CO}_{3}$ (上海国药， $\geqslant 99.8 \%$ )、 $\mathrm{Li}_{2} \mathrm{CO}_{3}$ (北京中

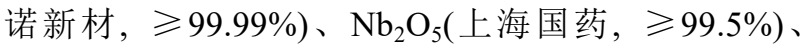
$\mathrm{Ta}_{2} \mathrm{O}_{5}$ (北京中诺新材, $\geqslant 99.99 \%$ ) 和 $\mathrm{Sb}_{2} \mathrm{O}_{3}$ (北京中诺新 材， $\geqslant 99.9 \%$ ) 的微粉为原料，通过固相反应制备 KNLNST 陶瓷样品。首先, 将烘干处理的原料按化学 计量比进行称量, 装入球磨罐中并添加适量的无水乙 醇进行 $16 \mathrm{~h}$ 的球磨。把球磨后的浆料烘干并压制成直 径为 $30 \mathrm{~mm}$ 的块体, 在 $930{ }^{\circ} \mathrm{C}$ 下预烧 $4 \mathrm{~h}$ 使原料之间 发生固相反应。将预烧的块体粉碎并再次球磨 $16 \mathrm{~h}$, 把浆料烘干得到所需的陶瓷粉。然后, 在陶瓷粉中加 入适量的聚乙烯醇粘合剂进行造粒, 在 $200 \mathrm{MPa}$ 的压 强下压制成直径 $15 \mathrm{~mm}$ 、厚度 $1.5 \mathrm{~mm}$ 左右的小圆片。 将小圆片在 $650{ }^{\circ} \mathrm{C}$ 温度条件下进行 $30 \mathrm{~min}$ 的排塑处理, 最后分别选择普通烧结方式或两步烧结方式制备得到 KNLNST 陶瓷样品。普通烧结时, 以 $3.5^{\circ} \mathrm{C} / \mathrm{min}$ 的升 温速率升至所需要的烧结温度, 保温 $5 \mathrm{~h}$ 后自然降 温。两步烧结时, 以 $10{ }^{\circ} \mathrm{C} / \mathrm{min}$ 的速率升温到 $T_{1}$ 并保 温 $1 \mathrm{~min}$, 然后快速降至 $T_{2}$ 进行 $4 \mathrm{~h}$ 的保温, 最后自然 降温。

为了表征介电性质和压电性能, 将烧结的陶瓷样 品的上下表面涂布银浆, 在 $570{ }^{\circ} \mathrm{C}$ 下进行 $30 \mathrm{~min}$ 的热 处理得到被覆银电极的陶瓷样品。使用 Agilent 4294 阻抗分析仪测试电容值 $C_{\mathrm{p}}$, 由计算得出相对介电常数 $\varepsilon^{\prime}$ 。对于压电性能的测试, 将陶瓷样品放置在 $45{ }^{\circ} \mathrm{C}$ 的 硅油中, 施加 $4 \mathrm{kV} / \mathrm{mm}$ 的直流电场进行 $30 \mathrm{~min}$ 的极化 处理。在室温下放置 $24 \mathrm{~h}$ 后, 用准静态压电系数测试 仪 YE2730A 测试压电系数 $d_{33}$ 。使用 Agilent 4294 阻 抗分析仪测试平面振动模式的谐振频率 $f_{\mathrm{r}}$ 和反谐振频 
率 $f_{\mathrm{a}}$, 计算得到机电耦合系数 $k_{\mathrm{p}}$ 。利用 D8 ADANCE X 射线衍射仪做物相分析，研究 KNLNST 陶瓷的晶体结 构。将表面镜面抛光的陶瓷样品在盐酸和氢氟酸的混 合水溶液中进行大约 $3 \mathrm{~min}$ 的酸腐蚀处理，利用扫描电 镜观察陶瓷表面的微观组织结构和电畴图案的形貌。

\section{2 结果与讨论}

表 1 给出了采用两种不同烧结方式制备的 KNLNST 陶瓷的密度 $\rho$ 、压电系数 $d_{33}$ 和平面机电耦合 系数 $k_{\mathrm{P}}$, 为了便于对实验结果进行描述, 在表 1 中分 别用 CS、TSS-1 等符号对各种不同烧结条件所制备的 KNLNST 陶瓷样品进行了编号。对于普通烧结方式, 研究首先对烧结温度进行了摸索, 确定的最佳烧结温 度为 $1090{ }^{\circ} \mathrm{C}$ 。此烧结条件下制备的 KNLNST 陶瓷的 密度 $\rho$ 为 $4.40 \mathrm{~g} / \mathrm{cm}^{3}$ 。利用 XRD 分析所得的晶格参数 计算出相应的相对密度 $\rho^{\prime}$ 为 $95.2 \%$ 。室温下测试得到的 $d_{33}$ 值为 $363 \mathrm{pC} / \mathrm{N} 、 k_{\mathrm{P}}$ 值为 0.48 。对于两步烧结方式, 研 究将第一步程序的烧结温度 $T_{1}$ 设置在 $1130 \sim 1160{ }^{\circ} \mathrm{C}$ 范 围、将第二步程序的烧结温度 $T_{2}$ 设置在 1040 1060 ${ }^{\circ} \mathrm{C}$ 范围进行了实验探索。结果表明, 烧结温度条件对制 备的 KNLNST 陶瓷的密度和压电性能有很大的影响。 当将 $T_{2}$ 设为 $1050{ }^{\circ} \mathrm{C}$ 、改变 $T_{1}$ 时, 随着 $T_{1}$ 的提高, 陶 瓷密度呈现先增大后减小的变化(推测可能是 $T_{1}$ 过高 时碱金属元素的挥发所致), 压电性能也相应地呈现先 增强后减弱的变化。如果将 $T_{1}$ 设为 $1150{ }^{\circ} \mathrm{C}$ 、改变 $T_{2}$ 进行实验, $T_{2}$ 为 $1040{ }^{\circ} \mathrm{C}$ 时的烧结效果不好, 而 $T_{2}$ 设为 1050 或 $1060{ }^{\circ} \mathrm{C}$ 时所制备出的 KNLNST 陶瓷比普通烧 结方式制备的 KNLNST 陶瓷有着更高的密度值。其中, $T_{2}$ 为 $1050{ }^{\circ} \mathrm{C}$ 条件下制备的 KNLNST 陶瓷呈现最高的 密度 $\rho=4.47 \mathrm{~g} / \mathrm{cm}^{3}$ (对应于 $\rho^{\prime}=97.0 \%$ ) 和最好的压电性能: $d_{33}=387 \mathrm{pC} / \mathrm{N} 、 k_{\mathrm{P}}=0.49$ 。总而言之, 在恰当的温度条件 进行两步烧结可以有效地提高 KNLNST 陶瓷的相对 密度和压电性能。从表 1 所示的实验结果也表明, 致 密度对 $\mathrm{KNN}$ 基陶瓷的 $d_{33}$ 值有很大的影响 ${ }^{[13-14,23]}$ 。

表 1 不同烧结条件下制备的 KNLNST 陶瓷的物理性能

Table 1 Physical property of KNLNST ceramics prepared under different sintering conditions

\begin{tabular}{ccccc}
\hline Sample & Sintering conditions & $\rho /\left(\mathrm{g} \cdot \mathrm{cm}^{-3}\right)$ & $d_{33} /\left(\mathrm{pC} \cdot \mathrm{N}^{-1}\right)$ & $k_{\mathrm{p}}$ \\
\hline $\mathrm{CS}$ & $1090{ }^{\circ} \mathrm{C}, 5 \mathrm{~h}$ & 4.40 & 363 & 0.48 \\
$\mathrm{TSS}-1$ & $1130{ }^{\circ} \mathrm{C} / 1050{ }^{\circ} \mathrm{C}, 4 \mathrm{~h}$ & 4.43 & 369 & 0.47 \\
$\mathrm{TSS}-2$ & $1140{ }^{\circ} \mathrm{C} / 1050{ }^{\circ} \mathrm{C}, 4 \mathrm{~h}$ & 4.44 & 370 & 0.43 \\
$\mathrm{TSS}-3$ & $1150{ }^{\circ} \mathrm{C} / 1040{ }^{\circ} \mathrm{C}, 4 \mathrm{~h}$ & 4.36 & 358 & 0.44 \\
$\mathrm{TSS}-4$ & $1150{ }^{\circ} \mathrm{C} / 1050{ }^{\circ} \mathrm{C}, 4 \mathrm{~h}$ & 4.47 & 387 & 0.49 \\
$\mathrm{TSS}-5$ & $1150{ }^{\circ} \mathrm{C} / 1060{ }^{\circ} \mathrm{C}, 4 \mathrm{~h}$ & 4.42 & 378 & 0.48 \\
TSS-6 & $1160{ }^{\circ} \mathrm{C} / 1050{ }^{\circ} \mathrm{C}, 4 \mathrm{~h}$ & 4.35 & 379 & 0.46 \\
\hline
\end{tabular}

图 1 所示的是各种不同烧结条件下制备的 KNLNST 陶瓷在室温下的 X 射线衍射图谱。图谱中所 有衍射峰均可以用钙钛矿型的噟立方晶体结构的一些 晶面指数标定, 不存在其他杂相所产生的衍射峰。图 1(b)是图 1(a)于 $2 \theta$ 为 $46.0^{\circ}$ 附近的衍射谱线的部分放 大，对应于赝立方晶体结构的 $\{200\}$ 晶面的一组衍射 峰。由该组衍射峰的谱线强度的比例关系可知, 所有 的 KNLNST 陶瓷室温下均处于正交相-四方相的两相 共存状态。

分别以普通烧结方式制备、编号为 CS 的 KNLNST 陶瓷(简称为 KNLNST-CS)和采用两步烧结方式在 $1150{ }^{\circ} \mathrm{C}, 1 \mathrm{~min}-1050{ }^{\circ} \mathrm{C}, 4 \mathrm{~h}$ 烧结条件下制备、编号为 TSS-4 的 KNLNST 陶瓷(简称为 KNLNST-TSS)为代表, 对其介电温谱和电畴结构进行详细的对比研究。图 2 所示的是两种代表性 KNLNST 陶瓷在 $2{ }^{\circ} \mathrm{C} / \mathrm{min}$ 的升温 过程中以 $1 \mathrm{kHz}$ 测试频率的条件下得到的 $\varepsilon^{\prime}$ 值随温度
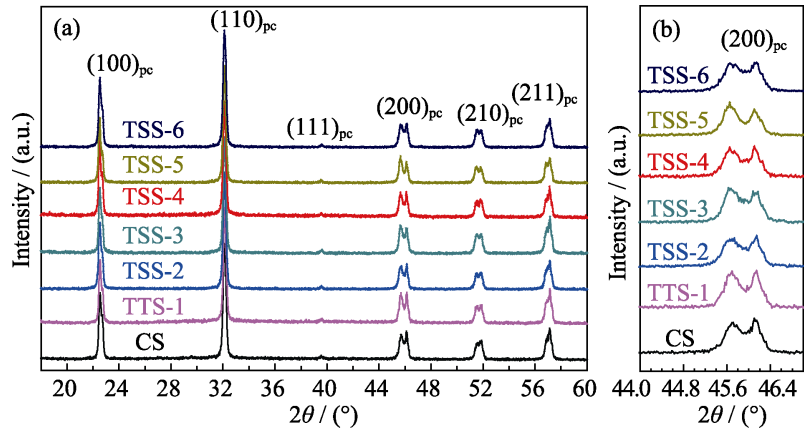

图 1 (a)各种未极化状态的 KNLNST 陶瓷于室温下的 XRD 图谱, (b) $2 \theta$ 为 $46.0^{\circ}$ 附近的部分放大谱图。

Fig. 1 (a) Room-temperature XRD patterns of the various unpoled KNN ceramics and (b) enlarged parts in (a)

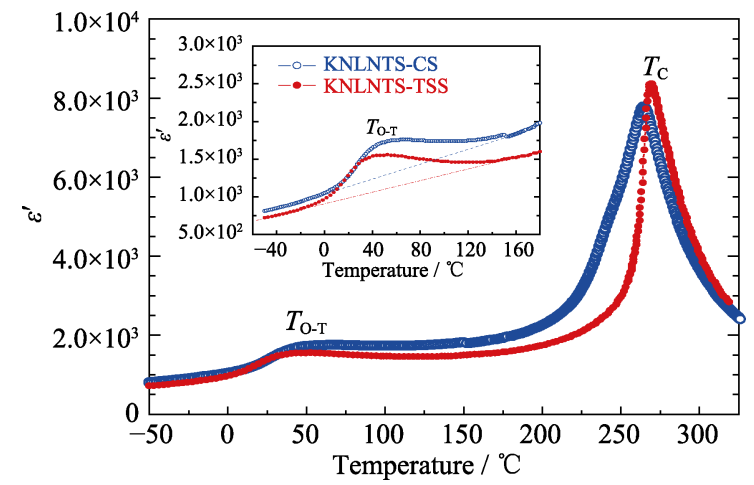

图 2 未极化状态的两种代表性 KNLNST 陶瓷在升温过程 中测试得到的介电温谱, 插图为正交相至四方相的相变的 介电温谱峰的部分放大图

Fig. 2 Temperature dependence of dielectric permittivity $\varepsilon^{\prime}$ of two representative unpoled KNLNST ceramics, prepared by conventional sintering and two-step sintering, respectively. Inset is partially enlarged curves of dielectric temperature peak of the phase transition from orthorhombic to tetragonal phase 
变化的曲线。介电温谱在 $-50{ }^{\circ} \mathrm{C}$ 至 $325{ }^{\circ} \mathrm{C}$ 的测试温度 区间呈现两个峰, 分别对应于正交晶相至四方相和四 方相至立方相的相变。将峰值温度分别标记为 $T_{\mathrm{O}-\mathrm{T}}$ 和 居里温度 $T_{\mathrm{C}}$, KNLNST-CS 陶瓷的 $T_{\mathrm{O}-\mathrm{T}}$ 和 $T_{\mathrm{C}}$ 分别约为 46 和 $265^{\circ} \mathrm{C}$, 而 KNLNST-TSS 陶瓷的 $T_{\mathrm{O}-\mathrm{T}}$ 和 $T_{\mathrm{C}}$ 分别 约为 39 和 $269{ }^{\circ} \mathrm{C}$ 。由此可见, 烧结方式对 KNLNST 陶瓷的 $T_{\mathrm{O}-\mathrm{T}}$ 值和 $T_{\mathrm{C}}$ 值有着一定的影响。相比于 KNLNST-CS 陶瓷, KNLNST-TSS 陶瓷的 $T_{\mathrm{O}-\mathrm{T}}$ 降低了大 约 $7{ }^{\circ} \mathrm{C}$, 而 $T_{\mathrm{C}}$ 则升高了 $4{ }^{\circ} \mathrm{C}$ 。由图 2 还可知, 正交相 至四方相的相变和四方相至立方相的相变均涉及到很 宽的温度区域。其中, 正交晶相至四方晶相的相变所 对应的介电峰的峰宽温度区域达 $150{ }^{\circ} \mathrm{C}$ (如图 2 插图所 示)。这一结果与上述 XRD 图谱所显示的两类 KNLNST 陶瓷室温下均处于正交相-四方相的两相共 存状态的现象是一致的。

图 3(a)是未极化状态的 KNLNST-CS 陶瓷的电畴 图案的典型扫描电镜照片, (b)是(a)中的虚线框中的部 分放大照片; 图 3(c) (d) 是未极化状态的 KNLNSTTSS 陶瓷的电畴图案的典型扫描电镜照片, 分别对应 着同一样品的不同位置。未极化状态的电畴图案乍看 比较杂乱, 晶界也不太容易辨别。通过仔细分析, 可以 发现其规律性, 即大多数的电畴图案是由平行条纹与 (无规则形状的)水痕状区域交叠而构成的。这些平行 条纹在三维空间中所对应的是片状的非 $180^{\circ}$ 电畴的叠

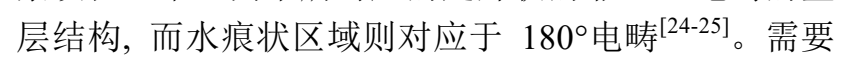
注意的是, 水痕状区域的内部存在着一些平行条纹。
这些无规则形状水痕的界面对应的是 $180^{\circ}$ 畴界; 水痕 状区域内部所包含的非 $180^{\circ}$ 电畴的总体极化与水痕状 区域外沿平行条纹方向的近邻区的总体极化的方向应 该是相反的。为了便于更好地理解, 在图 3(b)和(d)中 用白色小箭头符号指出了一些 $180^{\circ}$ 畴界或水痕状的 $180^{\circ}$ 电畴的位置。

此外, 对于 KNLNST-TSS 陶瓷, 如图 3(c)所示, 一些大晶粒内的部分电畴图案呈现微米级宽度的带状 区域, 其内部又包含着很多较短的平行条纹, 相邻带 状区域内的平行条纹间互成一定的角度。这种电畴图 案在文献中被称为鱼骨状结构的电畴构型 ${ }^{[24]}$ 。 KNLNST-CS 陶瓷的电畴图案的多数平行条纹宽度在 $170 \mathrm{~nm}$ 左右, KNLNST-TSS 陶瓷的电畴图案的多数平 行条纹宽度则小于 $100 \mathrm{~nm}$ 。换言之, 未极化的 KNLNST-TSS 陶瓷比未极化的 KNLNST-CS 陶瓷有更 高的电畴密度。

图 4(a)是经过极化处理的 KNLNST-CS 陶瓷的微 观组织结构和电畴图案的典型扫描电镜照片。对比 图 3 可知, 极化后的 KNLNST-CS 陶瓷的电畴图案相 对简单, 微观组织结构的晶界也变得清晰可辨。由图 4 可知, KNLNST-CS 陶瓷的晶粒径尺寸比较均一, 平均 尺寸大约为 $4 \mu \mathrm{m}$ 。晶粒内部的电畴图案一般由一组或 几组平行的条纹组成, 很多平行条纹贯穿晶粒, 平行 条纹的宽度分布在 $180 \mathrm{~nm}$ 至 $310 \mathrm{~nm}$ 之间。极化使水 痕状的 $180^{\circ}$ 电畴的极化取向发生翻转而消失, 使得非 $180^{\circ}$ 电畴的宽度有所增大。
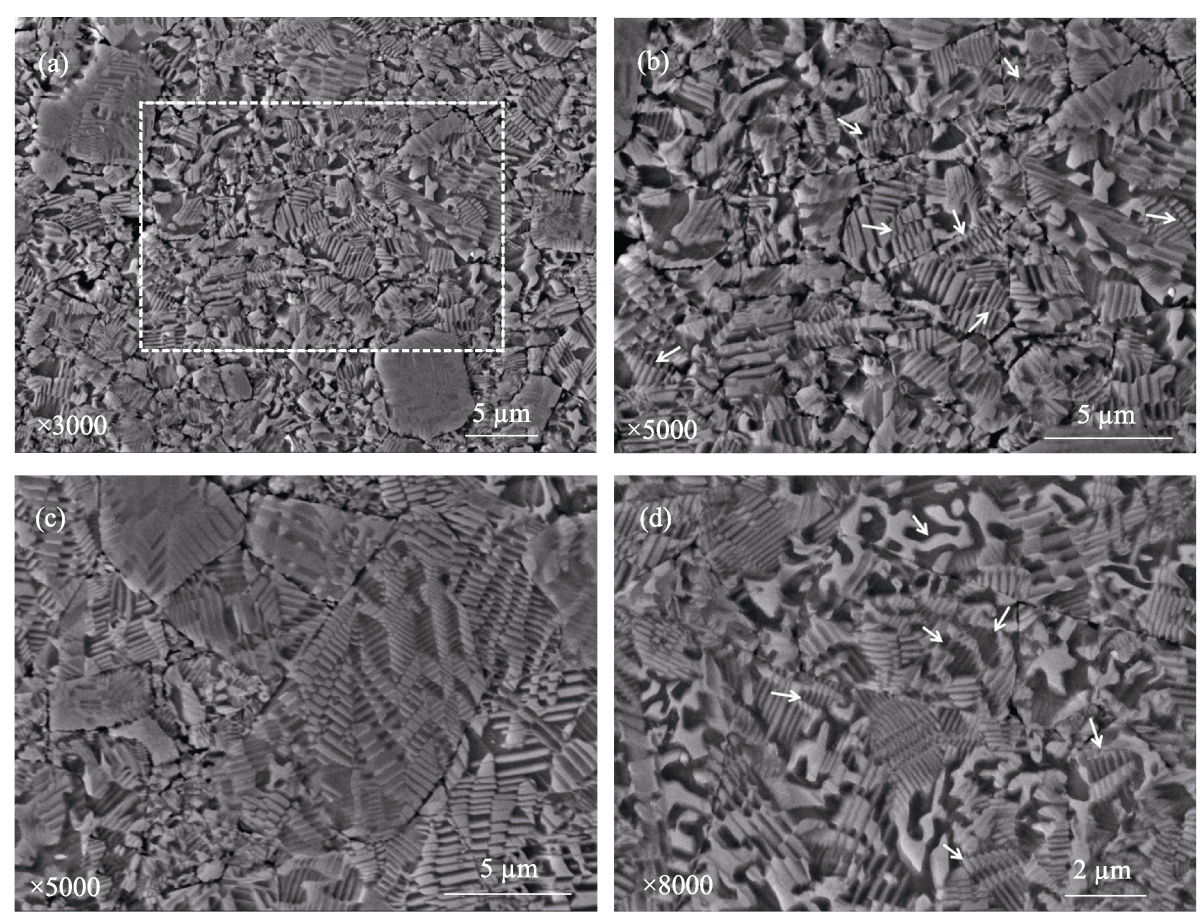

图 3 未极化状态的两种代表性 KNLNST 陶瓷的电畴图案的典型扫描电镜照片

Fig. 3 Typical SEM images of domain patterns observed in the two unpoled representative KNLNST ceramics (a) KNLNST-CS ceramic; (b) Partially enlarged view of (a); (c) and (d) KNLNST-TSS ceramic, corresponding to different locations of the same specimen 

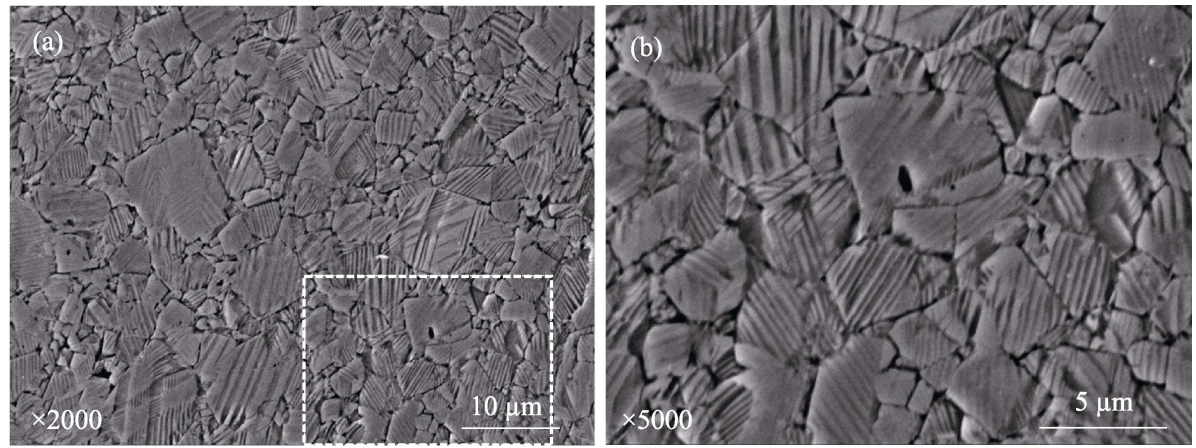

图 4 极化处理的 KNLNST-CS 陶瓷的微观组织结构和电畴图案的典型扫描电镜照片, (b) 是(a)的部分放大照片

Fig. 4 (a) Typical SEM image of microstructure and domain pattern of a poled KNLNST-CS ceramic,

(b) a partially enlarged image in (a)
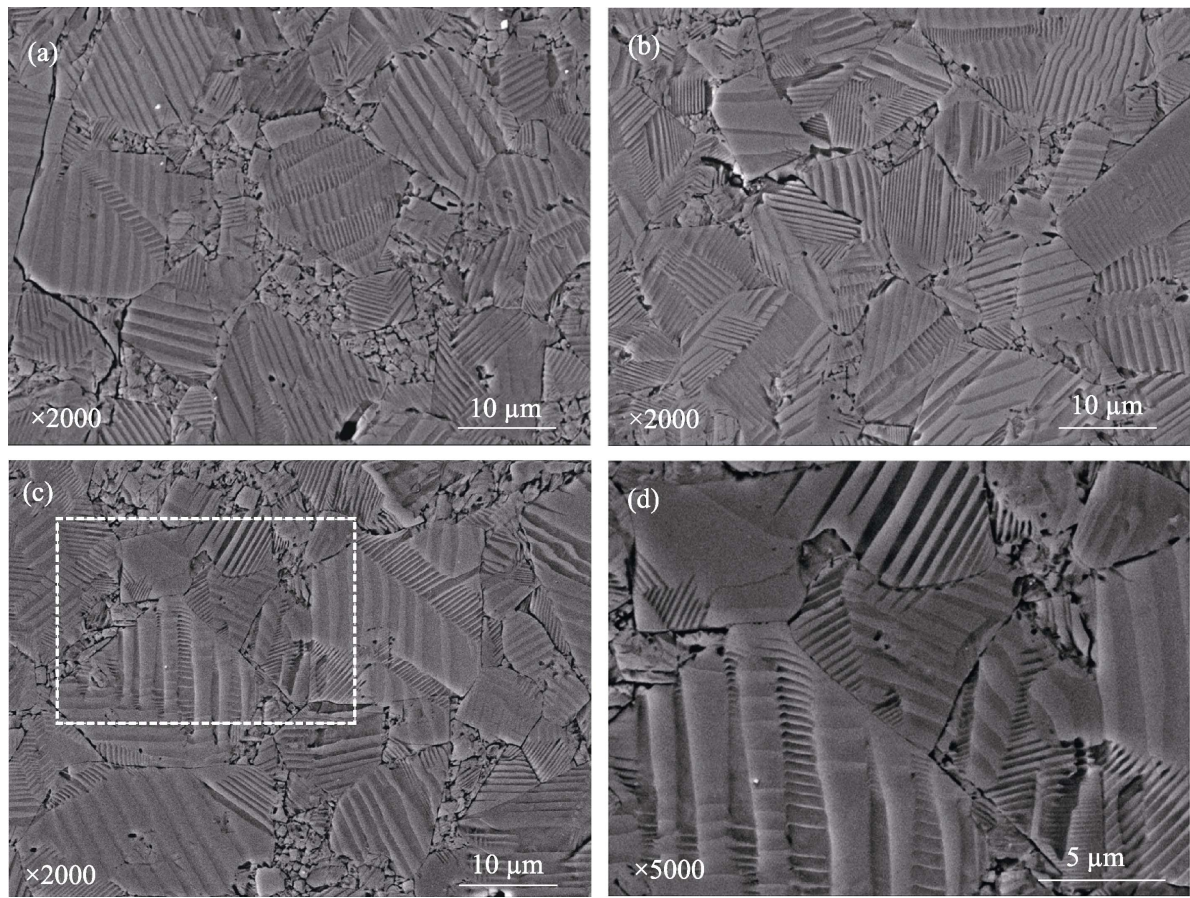

图 5 极化处理的 KNLNST-TSS 陶瓷的微观结构和电畴图案的典型扫描电镜照片

Fig. 5 Typical SEM images of microstructure and domain patterns observed in a poled KNLNST-TSS ceramic.

(a-c) Correspond to different positions of the same sample; (d) A partial enlarged image in (c)

图 5 是经过极化处理的 KNLNST-TSS 陶瓷的微观 组织结构和电畴图案的典型扫描电镜照片。KNLNSTTSS 陶瓷的晶粒尺寸分布不均匀，大晶粒之间镶嵌着 许多小晶粒，大晶粒径尺寸可达 $15 \mu \mathrm{m}$, 而小晶粒径 尺寸则在 $2 \mu \mathrm{m}$ 以下。从电畴图案中难以观察到水痕状 的 $180^{\circ}$ 电畴。除平行条纹的电畴图案之外, 很多大晶 粒中还存在着带状条纹, 其内部又包含着精细的平行 条纹结构。图 5(d)所示的高放大倍数的扫描电镜照片 清晰地显示了这种复杂的电畴结构。多数平行条纹的 宽度在 $200 \mathrm{~nm}$ 以上, 而带状条纹度的宽度则一般为数 微米, 其内部的精细平行条纹的宽度一般在 $140 \mathrm{~nm}$ 以 上。显然，与未极化状态的 KNLNST-TSS 陶瓷相比， 极化处理的 KNLNST-TSS 陶瓷的非 $180^{\circ}$ 电畴的宽度 增大了许多。

如上所述, KNLNST-CS 陶瓷和 KNLNST-TSS 陶
瓷在微观组织和电畴结构的两个方面有着很大的差 异。前者的致密度较低、晶粒径尺寸小但比较均匀, 后 者的致密度较高、晶粒径尺寸的大小分布不均匀。极 化处理的 KNLNST-CS 陶瓷的大多数晶粒呈现简单的 平行条纹的电畴图案, 而极化处理的 KNLNST-TSS 陶 瓷的许多大晶粒则呈现带状条纹中又存在着精细的平 行条纹的电畴图案。

\section{3 结论}

本研究分别采用普通烧结方式和两步烧结方式制 备 $\left(\mathrm{K}_{0.4425} \mathrm{Na}_{0.52} \mathrm{Li}_{0.0375}\right)\left(\mathrm{Nb}_{0.8825} \mathrm{Sb}_{0.08} \mathrm{Ta}_{0.0375}\right) \mathrm{O}_{3}$ 陶瓷，通 过 XRD 分析确定了室温下的晶体结构, 进行介电温谱 的测量考察了结构相变, 测试了压电系数 $d_{33}$ 和机电耦 合系数 $k_{\mathrm{P}}$, 利用抛光酸腐蚀方法观察了微观组织结构 
和电畴结构。研究发现:

1) 采取恰当烧结温度条件的两步烧结可以有效 地提高 KNLNST 陶瓷的致密度和压电性能。KNLNSTTSS 陶瓷的相对密度由 KNLNST-CS 陶瓷的 95.2\%增 加至 $97.0 \%, d_{33}$ 值由 $363 \mathrm{pC} / \mathrm{N}$ 增大到 $387 \mathrm{pC} / \mathrm{N}$ 。

2) 烧结方式对 KNLNST 陶瓷的微观组织结构和 电畴结构有着很大的影响。KNLNST-CS 陶瓷的晶粒 尺寸较小而且分布较为均匀, 极化处理后多数晶粒呈 现简单的平行条纹的电畴图案。KNLNST-TSS 陶瓷的 晶粒尺寸的大小分布不均匀, 极化处理后许多大晶粒 中呈现微米级的带状条纹, 带状条纹内又存在着精细 的平行条纹。

3) 两种烧结方式制备的 KNLNST 陶瓷室温下均 处于正交相和四方相的共存状态, 但烧结方式对 $T_{\mathrm{O}-\mathrm{T}}$ 值和 $T_{\mathrm{C}}$ 值有着一定的影响。KNLNST-TSS 陶瓷的 $T_{\mathrm{O}-\mathrm{T}}$ 和 $T_{\mathrm{C}}$ 分别约为 39 和 $269{ }^{\circ} \mathrm{C}$ 。与 KNLNST-CS 陶瓷相 比较, KNLNST-TSS 陶瓷的 $T_{\mathrm{O}-\mathrm{T}}$ 降低了大约 $7{ }^{\circ} \mathrm{C}$, 而 $T_{\mathrm{C}}$ 则升高了 $4{ }^{\circ} \mathrm{C}$ 。

\section{参考文献:}

[1] GAO Y, ZHANG J L, ZONG X J, et al. Extremely temperaturestable piezoelectric properties of orthorhombic phase in $(\mathrm{K}, \mathrm{Na}) \mathrm{NbO}_{3^{-}}$ based ceramics. J. Appl. Phys., 2010, 107(7): 074101-1-5.

[2] SAITO Y, TAKAO H, TANI T, et al. Lead-free piezoceramics. Nature, 2004, 432(4): 84-87.

[3] GUO Y P, KAKIMOTO K, OHSATO H. Phase transitional behavior and piezoelectric properties of $\left(\mathrm{Na}_{0.5} \mathrm{~K}_{0.5}\right) \mathrm{NbO}_{3}-\mathrm{LiNbO}_{3}$ ceramics. Appl. Phys. Lett., 2004, 85(18): 4121-4123.

[4] GUO Y P, KAKIMOTO K, OHSATO H. $\left(\mathrm{Na}_{0.5} \mathrm{~K}_{0.5}\right) \mathrm{NbO}_{3}-\mathrm{LiTaO}_{3}$ lead-free piezoelectric ceramics. Mater. Lett., 2005, 59(2/3): 241-244.

[5] FU J, ZUO R Z, WANG X H, et al. Polymorphic phase transition and enhanced piezoelectric properties of $\mathrm{LiTaO}_{3}$-modified $\left(\mathrm{Na}_{0.52} \mathrm{~K}_{0.48}\right)\left(\mathrm{Nb}_{0.93} \mathrm{Sb}_{0.07}\right) \mathrm{O}_{3}$ lead-free ceramics. J. Phys. D: Appl. Phys., 2009, 42(1): 012006-1-4.

[6] GAO Y, ZHANG J L, QIN Y L, et al. Remarkably strong piezoelectricity of lead-free $\left(\mathrm{K}_{0.45} \mathrm{Na}_{0.55}\right)_{0.98} \mathrm{Li}_{0.02}\left(\mathrm{Nb}_{0.77} \mathrm{Ta}_{0.18} \mathrm{Sb}_{0.05}\right) \mathrm{O}_{3}$ ceramic. J. Am. Ceram. Soc., 2011, 94(9): 2968-2973.

[7] ZHANG B Y, WU J G, CHENG X J, et al. Lead-free piezoelectrics based on potassium-sodium niobate with giant $d_{33}$. ACS Appl. Mater. Interfaces, 2013, 5(16): 7718-7725.

[8] WANG X P, WU J G, XIAO D Q, et al. Giant piezoelectricity in potassium-sodium niobate lead-free ceramics. J. Am. Chem. Soc., 2014, 136(7): 2905-2910.

[9] ZHENG T, WU J G, CHEN Q, et al. Composition-driven phase boundary and piezoelectricity in potassium-sodium niobate-based ceramics. ACS Appl. Mater. Interfaces, 2015, 7(36): 20332-20341.

[10] WU B, WU H J, WU J G, et al. Giant piezoelectricity and high Curie temperature in nanostructured alkali niobate lead-free piezoceramics through phase coexistence. J. Am. Chem. Soc., 2016, 138(47): 15459-15464.

[11] XU K, LI J, LÜ X, et al. Superior piezoelectric properties in potassiumsodium niobate lead-free ceramics. Adv. Mater, 2016, 28(38): 8519-8523.

[12] ZHANG M H, WANG K, DU Y J, et al. High and temperatureinsensitive piezoelectric strain in alkali niobate lead-free perovskite. J. Am. Chem. Soc., 2017, 139(10): 3889-3895.

[13] JAEGER E, EGERTON L. Hot pressing of potassium-sodium niobates. J. Am. Ceram. Soc., 1962, 45(5): 209-213.

[14] ZHANG S J, LEE H J, MA C, et al. Sintering effect on microstructure and properties of $(\mathrm{K}, \mathrm{Na}) \mathrm{NbO}_{3}$ ceramics. J. Am. Ceram. Soc., 2011, 94(11): 3659-3665.

[15] LI J F, WANG K, ZHANG B P, et al. Ferroelectric and piezoelectric properties of fine-grained $\mathrm{Na}_{0.5} \mathrm{~K}_{0.5} \mathrm{NbO}_{3}$ lead-free piezoelectric ceramics prepared by spark plasma sintering. J. Am. Ceram. Soc., 2006, 89(2): 706-709.

[16] ERIKSON M, YAN H X, VIOLA G, et al. Ferroelectric domain structures and electrical properties of fine-grained lead-free sodium potassium niobate ceramics. J. Am. Ceram. Soc., 2011, 94(10): 3391-3396.

[17] CHEN I W, WANG H X. Sintering dense nanocrystalline ceramics without final-stage grain growth. Nature, 2000, 404(9): 168-71.

[18] WANG X H, CHEN P L, CHEN I W. Two-step sintering of ceramics with constant grain-size. J. Am. Ceram. Soc., 2006, 89(2): 431-437.

[19] WANG X H, DENG X Y, BAI H L, et al. Two-step sintering of ceramics with constant grain-size, $\mathrm{II}_{\mathrm{BaTiO}} \mathrm{Ba}_{3}$ and $\mathrm{Ni}-\mathrm{Cu}-\mathrm{Zn}$ ferrite. J. Am. Ceram. Soc., 2006, 89(2): 438-443.

[20] FANG J, WANG X H, TIAN Z B, et al. Two-step sintering: an approach to broaden the sintering temperature range of alkaline niobate-based lead-free piezoceramics. J. Am. Ceram. Soc., 2010, 93(11): 3552-3555.

[21] PANG X M, QIU J H, ZHU K J, et al. (K,Na) $\mathrm{NbO}_{3}$-based lead-free piezoelectric ceramics manufactured by two-step sintering. Ceram. Int., 2012, 38(3): 2521-2527.

[22] WU J G, WANG Y M. Two-step sintering of new potassium sodium niobate ceramics: a high $d_{33}$ and wide sintering temperature range. Dalton Trans., 2014, 43(34): 12836-12841.

[23] ZHANG J L, QIN Y L, GAO Y, et al. Improvement of physical properties for KNN-based ceramics by modified two-step sintering. J. Am. Ceram. Soc., 2014, 97(3): 759-764.

[24] ARLT G, SASKO P. Domain configuration and equilibrium size of domains in $\mathrm{BaTiO}_{3}$ ceramics. J. Appl. Phys., 1980, 51(9): 4956- 496.

[25] JUAREZ R L, PERALTA O N, GARCÍA F G, et al. Ferroelectric domain structure of lead-free potassium-sodium niobate ceramics. J. Eur. Ceram. Soc., 2011, 31(9): 1861-1864. 